$13^{\text {th }}$ International Conference on AEROSPACE SCIENCES \& AVIATION TECHNOLOGY, ASAT- 13, May 26 - 28, 2009, E-Mail: asat@mtc.edu.eg Military Technical College, Kobry Elkobbah, Cairo, Egypt Tel : +(202) $24025292-24036138$, Fax: +(202) 22621908

\title{
Double Stage Controller for High Flexible Link Manipulators
}

\author{
Eyad Sh. Jabbour ${ }^{*}$, Hussein M. Mahgoub ${ }^{* *}$, Nabil G. Mekhail ${ }^{* *}$, Ahmed M. A. Elraouf ${ }^{* * *}$
}

\begin{abstract}
It is possible to use a PD controller to get acceptable tracking to a joint angle trajectory of a single flexible link manipulator. However, it has a poor capability to damp out end effector vibrations at the end of the trajectory following phase. Another controller can be used at the end of this phase to damp out end effector vibrations excited during this phase. The paper suggests the start of application of the second controller at the midpoint of the trajectory phase, and not at its end. This idea under consideration is investigated by a SIMULINK model within MATLAB environment where better results concerning overshoot are obtained.
\end{abstract}

Keywords: Flexible manipulator, robot control, model based controller.

\section{Introduction}

Smaller mass, lower peak power and less energy consumption are among the main potential advantages of Flexible Link Manipulators (FLM) over rigid link manipulators. Because of these potential advantages, their application in industry is expected to increase providing that their performance becomes more predictable and reliable. To improve the general performance of FLM, many researches have been carried out during the last decades. A considerable portion of these researches have been focused on End-Effecor Trajectory Tracking (EETT) because of its importance. In recent decade, much attention has been paid to modeling and control of flexible manipulators. This is motivated by the need for space-based manipulators that are necessarily lightweight and therefore flexible.

Due to high transportation cost, the expense of large motor and amplifiers required to derive massive earth-based industrial manipulators is an additional motivation for the design and control of lightweight manipulators. Furthermore, even for robot manipulators normally considered rigid links, flexibility cannot be neglected during fast motion. In this situation, elastic deformation usually causes vibration of manipulator, so the maneuvering time and accuracy are limited.

The dynamic equations of flexible manipulators are strongly coupled and highly nonlinear such that elastic deformation and the completely rigid rotation are coupled. Since 1970s, W. J. Book et al. began to study the dynamic model and the control of flexible manipulator [1]. A great extensive investigation has been done in the first decade and exemplified by the widely cited references such as [2].

\footnotetext{
${ }^{*}$ Syrian Armed Forces

** Egyptian Armed Forces

${ }^{* * *}$ Prof. Dr., Menoufia University
} 
Many control schemes have been proposed for flexible manipulators. Linear control approaches were used for the point to point motion control of a single flexible robot link based on the linear-quadratic-Gaussian (LQG) control method [3]. Acceleration feedback is used for the end point position control of the flexible arm. In this paper, what is called "model based control scheme" is used. The response for high acceleration or small rigidity of robot links is developed. The results are compared using a SIMULINK model in MATLAB for a case study of one flexible link manipulator without taking the gravity effect into consideration. The proposed modification can significantly enhance the performance of the trajectory tracking when undergoing high acceleration concerning max overshoot.

\section{Modeling}

Once the total kinetic and potential energies are obtained, Lagrange equations are used to derive the equations of motion of flexible manipulators [4]. The equations of motion can be written in a compact matrix form as

$\left(\begin{array}{ll}M_{\mathrm{rr}} & \mathrm{M}_{\mathrm{rf}} \\ \mathrm{M}_{\mathrm{rf}} & \mathrm{M}_{\mathrm{ff}}\end{array}\right)\left(\begin{array}{l}\ddot{\mathrm{q}}_{\mathrm{r}} \\ \ddot{\mathrm{q}}_{\mathrm{f}}\end{array}\right)+\left(\begin{array}{l}\mathrm{C}_{\mathrm{r}}(\mathrm{q}, \dot{\mathrm{q}}) \\ \mathrm{C}_{\mathrm{f}}(\mathrm{q}, \dot{\mathrm{q}})\end{array}\right)+\left(\begin{array}{c}\mathrm{G}_{\mathrm{r}}(\mathrm{q}) \\ \mathrm{G}_{\mathrm{f}}(\mathrm{q})\end{array}\right)+\left(\begin{array}{cc}0 & 0 \\ 0 & {[\mathrm{~K}]}\end{array}\right)\left(\begin{array}{l}\mathrm{q}_{\mathrm{r}} \\ \mathrm{q}_{\mathrm{f}}\end{array}\right)=\left(\begin{array}{l}\tau \\ 0\end{array}\right)$

where the vector of generalized variables $q$, consists of an $n \times 1$ of rigid joint variables $q_{r}$ together with an $N \times 1$ vector of flexible variables $q_{f}$. The mass matrix consists of an $n \times n$ symmetric, positive definite sub-matrix associated with the second derivative of the rigid joint variables or $\ddot{q}_{r}$ and is denoted by $\left[M_{r r}\right]$. The $N \times N$ sub matrix $\left[M_{f f}\right]$ is also symmetric, positive definite matrix and is associated with the second derivative of the flexible variables of $\ddot{q}_{f}$. The sub matrix $M_{r f}$ is $N \times n$ and represents the coupling between the rigid joint variables and the elastic variables in the link. $C_{r}(q, \dot{q}), G_{r}(q) C_{f}(q, \dot{q})$ and $G_{f}(q)$ represent the Coriolis and centripetal terms and the gravity terms for rigid degrees of freedom and flexible ones respectively. The $N \times N$ symmetric, positive definite matrix $[K]$ is called the flexural stiffness matrix and arises from strain energy of the flexible links. The right hand side of the equations of motion contains a $n \times 1$ vector of input torques applied at the joints.

\section{Control of Flexible Link Manipulators}

The dynamic equations of motion of flexible manipulator, Eq.(1), show the coupling between the rigid variables $q_{r}$ and the flexible variables $q_{f}$. The input torques will not only result in gross motion of the links but also the coupling will lead to transverse vibrations. This is shown schematically for a single link flexible manipulator in the block diagram of Fig. 1.

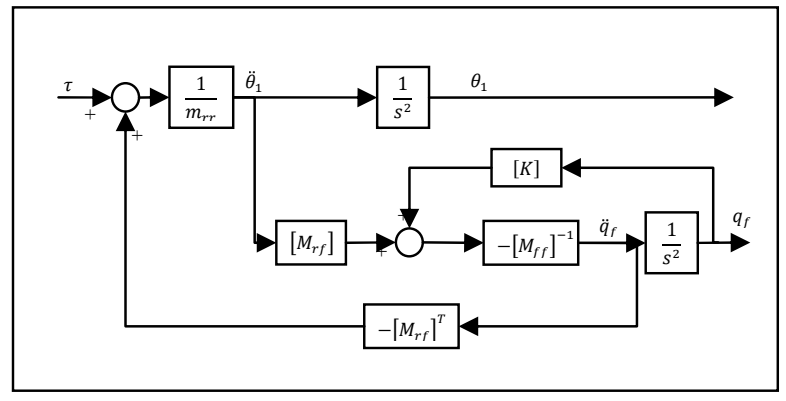

Fig. 1 Block diagram of a single flexible link manipulator. 


\section{Model Based Control for Trajectory Following}

To develop a model based control for a flexible link manipulator system, we start by rewriting the equations of motion (1), are rewritten as follows

$$
\begin{aligned}
& {\left[\mathrm{M}_{\mathrm{rr}}\right] \ddot{\mathrm{q}}_{\mathrm{r}}+\left[\mathrm{M}_{\mathrm{rf}}\right]^{\mathrm{T}} \ddot{\mathrm{q}}_{\mathrm{f}}+\mathrm{C}_{\mathrm{r}}(\mathrm{q}, \dot{\mathrm{q}})+\mathrm{G}_{\mathrm{r}}(\mathrm{q})=\tau} \\
& {\left[\mathrm{M}_{\mathrm{rf}}\right] \ddot{\mathrm{q}}_{\mathrm{r}}+\left[\mathrm{M}_{\mathrm{ff}}\right] \ddot{\mathrm{q}}_{\mathrm{f}}+\mathrm{C}_{\mathrm{f}}(\mathrm{q}, \dot{\mathrm{q}})+\mathrm{G}_{\mathrm{f}}(\mathrm{q})+[\mathrm{K}] \mathrm{q}_{\mathrm{f}}=0}
\end{aligned}
$$

Solving for $\ddot{q}_{f}$ then

$$
\ddot{q}_{f}=-\left[M_{f f}\right]^{-1}\left(\left[M_{r f}\right] \ddot{q}_{r}+C_{f}+G_{f}+[K] q_{f}\right)
$$

Substituting $\ddot{q}_{f}$ in Eqn (2), then

$$
\begin{gathered}
\left(\left[M_{r r}\right]-\left[M_{r f}\right]^{T}\left[M_{f f}\right]^{-1}\left[M_{r f}\right]\right) \ddot{q}_{r}+\left\{C_{r}+G_{r}-\left[M_{r f}\right]^{T}\left[M_{f f}\right]^{-1}\left(C_{f}+G_{f}+[K] q_{f}\right)\right\} \\
=\tau
\end{gathered}
$$

Now assuming that the output of a controller can be written as

$\tau_{q r}=[\alpha] \tau_{q r}+\beta$

Where

$$
\begin{aligned}
& {[\alpha]=\left(\left[M_{r r}\right]-\left[M_{r f}\right]^{T}\left[M_{f f}\right]^{-1}\left[M_{r f}\right]\right)} \\
& \beta=C_{r}+G_{r}-\left[M_{r f}\right]^{T}\left[M_{f f}\right]^{-1}\left(C_{f}+G_{f}+[K] q_{f}\right)
\end{aligned}
$$

With $q_{r d}(t)$ denoting the desired joint trajectory. The linear error equation for the unit inertia plant is then deduced

$\ddot{e}_{r}(t)+[K p]_{q r} e_{r}(t)+\left[K_{v}\right]_{q r} \dot{e}_{r}(t)=0$

where $e_{r}(t)=q_{r d}-q_{r}$

The appropriate choice of controller gains $[\mathrm{Kp}]_{\mathrm{qr}}$ and $\left[\mathrm{K}_{\mathrm{v}}\right]_{\mathrm{qr}}$, ensures that $e_{r}(t)$ tends to zero asymptotically and the controller can track any desired joint trajectory.

To study the stability of the resulting flexible oscillations, the zero dynamics (when inputs are chosen to constrain the output of the system to be zero or constant, Isidori1989) is given by:

$\ddot{q}_{f}=-\left[M_{f f}\right]^{-1}\left(C_{f}+G_{f}+[K] q_{f}\right)$

where all terms are evaluated for a constant $q_{r}^{*}$ and for $\dot{q}_{r}=0$.

The equilibrium points of the system are given by $\dot{q}_{f}=0$ and a $q_{f}^{*}$, which satisfies

$[K] q_{f}^{*}+G_{f}\left(q_{r}^{*}, q_{f}^{*}\right)=0$

To study the stability, the following Lyapunov function is used [5] 


$$
\begin{aligned}
V\left(q_{f}, \dot{q}_{f}\right)= & \frac{1}{2} \dot{q}_{f}^{T}\left[M_{f f}\right] \dot{q}_{f}+\frac{1}{2}\left(q_{f}^{*}-q_{f}\right)^{T}[K]\left(q_{f}^{*}-q_{f}\right)+\left[V_{G}\left(q_{r}^{*}, q_{f}\right)-V_{G}\left(q_{r}^{*}, q_{f}^{*}\right)\right] \\
& +\left(q_{f}^{*}-q_{f}\right)^{T} G_{f}\left(q_{r}^{*}, q_{f}^{*}\right)
\end{aligned}
$$

By differentiation with respect to time, it gives

$\dot{V}\left(q_{f}, \dot{q}_{f}\right)=0$

This implies that, the zero dynamics of the resulting closed loop system is not asymptotically stable, but only critically stable. However, if material damping is incorporated in the model, the zero dynamics of the system can become asymptotically stable, which is naturally satisfied. The control scheme described above uses the notion of feedback linearization and this is very different from linearization of a nonlinear system.

\section{Tip Position Vibration Control}

The control scheme for end position vibration control is based on sensing the vibration of the end-effector and using the rigid Jacobean matrix of a flexible manipulator. A controller based on the measured $\mu$ and $\dot{\mu}$ and with gravity compensation can be written as

$\tau_{\mu}=\left[J_{q r}^{r}\right]^{T}\left(-\left[K_{p}\right]_{\mu} \delta \mu-\left[K_{v}\right]_{\mu} \dot{\mu}\right)+G_{r}\left(q_{r d}, q_{f d}\right)$

Where $\mu$ is the $6 \times 1$ entity representing the position and orientation of the end-effector, $\dot{\mu}$ is the $6 \times 1$ entity representing the axial and angular velocity of the end-effector, $\delta \mu$ is the quantity $\mu-\mu_{d}$ and is the error between the measured end effector position and the desired end effector position. The gain matrices $\left[K_{p}\right]_{\mu}$ and $\left[K_{v}\right]_{\mu}$ are constant diagonal matrices representing the position and velocity gains, respectively.

To analyze the stability of the equilibrium point, the following Lyabunov function is considered.

$$
\begin{gathered}
V=\frac{1}{2} \dot{q}^{T}[M(q)] \dot{q}+\frac{1}{2}\left(q_{f d}-q_{f}\right)^{T}[K]\left(q_{f d}-q_{f}\right)+\left[V_{G}(q)-V_{G}\left(q_{d}\right)\right] \\
+\left(q_{d}-q\right)^{T} G\left(q_{d}\right)+\frac{1}{2} \delta \mu^{T}\left[K_{p}\right]_{\mu} \delta \mu
\end{gathered}
$$

The time derivation of $V$ along the trajectories of the closed loop system yields that the minimum eigenvalue of the velocity feedback gain matrix can be chosen to ensure stability $(\dot{V}<0),[5]$.

\section{A Double-Stage Control Algorithm}

The control law based on the Eqns (5),(6)and (7) can achieve asymptotic trajectory following for the rigid joint variables $q_{r}$. It, however, has poor capability to damp out end-effector vibrations at the end of the trajectory following phase. The end position vibration control law given in Eqn (13) can be used after the trajectory following phase to damp out end-effector vibrations excited during this phase. So, a double Stage Controller (DCLR) is considered. It is given by 


$$
\tau=([\mathrm{U}]-[\mathrm{S}]) \tau_{\mathrm{qr}}+[\mathrm{S}] \tau_{\mu}
$$

where

$$
[S]=\left\{\begin{array}{l}
{[0] \text { null matrix during joint trajectory tracking stage }} \\
{[\mathrm{U}] \text { identity matrix during end position vibration control }}
\end{array}\right\}
$$

$$
\begin{aligned}
& \tau_{\mathrm{qr}}=\left(\left[\mathrm{M}_{\mathrm{rr}}\right]-\left[\mathrm{M}_{\mathrm{rf}}\right]^{\mathrm{T}}\left[\mathrm{M}_{\mathrm{ff}}\right]^{-1}\left[\mathrm{M}_{\mathrm{rf}}\right]\right) \epsilon_{\mathrm{qr}}+\mathrm{C}_{\mathrm{r}}+\mathrm{G}_{\mathrm{r}}-\left[\mathrm{M}_{\mathrm{rf}}\right]^{\mathrm{T}}\left[\mathrm{M}_{\mathrm{ff}}\right]^{-1}\left(\mathrm{C}_{\mathrm{f}}+\mathrm{G}_{\mathrm{f}}+[\mathrm{K}] \mathrm{q}_{\mathrm{f}}\right) \\
& \tau_{\mu}=\left[\mathrm{J}_{\mathrm{qr}}^{\mathrm{r}}\right]^{\mathrm{T}}\left(-\left[\mathrm{K}_{\mathrm{p}}\right]_{\mu} \delta \mu-\left[\mathrm{K}_{\mathrm{v}}\right]_{\mu} \dot{\mu}\right)+\mathrm{G}_{\mathrm{r}}\left(\mathrm{q}_{\mathrm{rd}}, \mathrm{q}_{\mathrm{fd}}\right)
\end{aligned}
$$

The block diagram representation of a double stage controller is shown in Fig. 2.

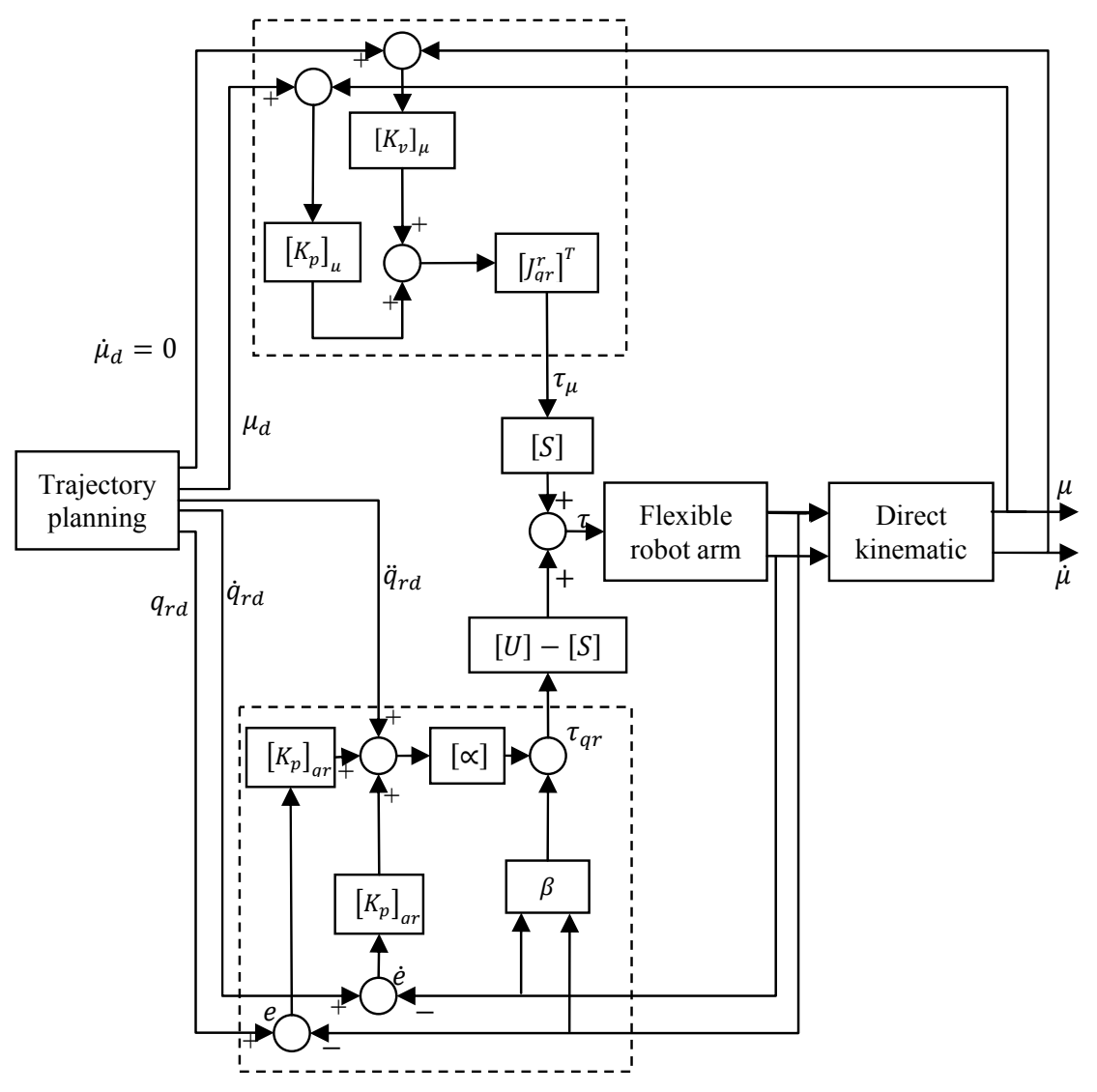

Fig. 2 Block diagram of the DCLR for single flexible link manipulator.

For the discussion of the suggested Midpoint Double Stage Controller (MP-DCLR), the same previous procedure is followed except for $[\mathrm{S}]$ which needs to be modified as follows:

$$
[S]=\left\{\begin{array}{l}
{[0] \text { null matrix during first half of joint trajectory tracking stage }} \\
{[\mathrm{U}] \text { identity matrix at the moment corresponds to midpoint of trajectory }}
\end{array}\right\}
$$




\section{The Case Study}

In this work, a single link flexible manipulator is considered. Its block diagram is shown in Fig. 1. And in addition to the rigid angle at the joint hub, another angle, "the tip angle" is chosen to express link tip deflection such that

$$
\theta_{\text {tip }}=\theta_{r}+w(L, t) / L
$$

where $\theta_{\text {tip }}$ is tip angle measured at joint, $\mathrm{L}$ is link length and $w(L, t)$ is tip deflection, Fig. 3.

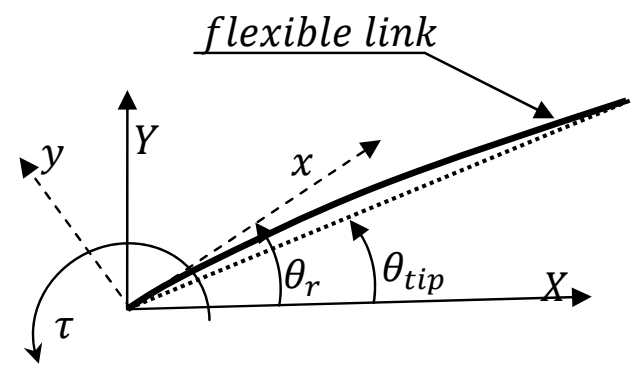

Fig. 3 The parameters of flexible manipulator system.

where XOYand xoy represent the stationary and moving coordinates frames respectively, $\tau$ represents the applied torque at the hub. $\theta(\mathrm{t})$ represent. In this study, a steel type flexible manipulator of dimensions $307 \times 26 \times 1 \mathrm{~mm}^{3}$, is considered with the following specifications

Table 1 Flexible robot specifications.

\begin{tabular}{|l|c|l|}
\hline $\mathrm{E}:$ & $2 \mathrm{e} 11 \mathrm{~N} / \mathrm{m}^{2}$ & Young modulus \\
\hline $\mathrm{I}:$ & $2.1667 \mathrm{e}-012 \mathrm{~m}^{4}$ & Area moment of inertia \\
\hline$\rho:$ & $7800 \mathrm{~kg} / \mathrm{m}^{3}$ & Mass density \\
\hline $\mathrm{a}:$ & $2.6000 \mathrm{e}-005 \mathrm{~m}^{2}$ & Cross-sectional area \\
\hline $\mathrm{L}:$ & $0.307 \mathrm{~m}$ & Length of the link \\
\hline
\end{tabular}

Considering the link consists of two flexible elements with 0.1 material damping coefficient, the following numerical values are obtained using the Finite Elements approach:

$$
\begin{aligned}
& M_{f f}=\left[\begin{array}{cc}
0.023124994285714 & 0.000000000000000 \\
0.000000000000000 & 0.000013971202477 \\
0.004002402857143 & 0.000147903609286 \\
-0.000147903609286 & -0.00000523920092
\end{array}\right. \\
& M_{r f}=\left[\begin{array}{c}
0.0047784243 \\
0.0000488992 \\
0.0040616607 \\
-0.0000977984
\end{array}\right] \\
& M_{r r}=[0.001955968346800] \\
& K=\left[\begin{array}{lllcl}
0 & 0 & 0 & 0 & 0 \\
0 & 2875.47 & 0 & -1437.73 & 110.35 \\
0 & 0 & 22.58 & -110.35 & 5.64 \\
0 & -1437.73 & -110.35 & 1437.73 & -110.34 \\
0 & 110.34 & 5.64 & -110.34 & 11.29
\end{array}\right]
\end{aligned}
$$$$
0.004002402857143-0.000147903609286
$$$$
0.000147903609286-0.000005239200929
$$$$
0.011562497142857 \quad-0.000250298415714
$$ 
The two controllers are applied to control the flexible link behavior by determining the actual torque applied at the motor joint so that the tip angle follows the joint angle. The major factor that dominates the tracking process is the acceleration. In this work the time dedicated for the robot to make $180^{\circ}$ of joint movement is fixed which means that less angle value has smaller period of time to perform and consequently, higher acceleration and deceleration at the beginning and end of motion respectively. In other words, smaller angle means larger acceleration, which expressed here by means of what so called position factor P.F. which will be explained later in this paper.

The state space of the previous model can be described by

$\dot{\mathrm{X}}=\mathrm{AX}+\mathrm{B}$

$\mathrm{Y}=\mathrm{CX}$

where:

$$
\begin{aligned}
& \mathrm{A}=\left(\begin{array}{llllllllll}
0 & 1 & 0 & 0 & 0 & 0 & 0 & 0 & 0 & 0 \\
0 & 0 & 20760728 & 22397 & -5047371 & -2405 & 24879024 & 22661 & -1895238 & -1195 \\
0 & 0 & 0 & 1 & 0 & 0 & 0 & 0 & 0 & 0 \\
0 & 0 & -3740992 & -3776 & 764643 & 363 & -3455222 & -3340 & 255978 & 166 \\
0 & 0 & 0 & 0 & 0 & 1 & 0 & 0 & 0 & 0 \\
0 & 0 & -29787131 & -20969 & -890712 & -517 & 18560542 & 4159 & -1618127 & -686 \\
0 & 0 & 0 & 0 & 0 & 0 & 0 & 1 & 0 & 0 \\
0 & 0 & -7759096 & -7504 & 1417882 & 673 & -5819322 & -5999 & 397007 & 272 \\
0 & 0 & 0 & 0 & 0 & 0 & 0 & 0 & 0 & 1 \\
0 & 0 & -104706281 & -58730 & -5146339 & -2642 & 96356858 & 42942 & -9718664 & -4750
\end{array}\right) \\
& B=\left[\begin{array}{c}
0 \\
2.6651 \\
0 \\
-4205 \\
0 \\
-1.3431 \\
0 \\
-0.8036 \\
0 \\
1.3935
\end{array}\right] \quad \mathrm{C}=\left(\begin{array}{llllllllll}
1 & 0 & 0 & 0 & 0 & 0 & 0 & 0 & 0 & 0 \\
0 & 1 & 0 & 0 & 0 & 0 & 0 & 0 & 0 & 0 \\
0 & 0 & 1 & 0 & 0 & 0 & 0 & 0 & 0 & 0 \\
0 & 0 & 0 & 1 & 0 & 0 & 0 & 0 & 0 & 0 \\
0 & 0 & 0 & 0 & 1 & 0 & 0 & 0 & 0 & 0 \\
0 & 0 & 0 & 0 & 0 & 1 & 0 & 0 & 0 & 0 \\
0 & 0 & 0 & 0 & 0 & 0 & 1 & 0 & 0 & 0 \\
0 & 0 & 0 & 0 & 0 & 0 & 0 & 1 & 0 & 0 \\
0 & 0 & 0 & 0 & 0 & 0 & 0 & 0 & 1 & 0 \\
0 & 0 & 0 & 0 & 0 & 0 & 0 & 0 & 0 & 1
\end{array}\right)
\end{aligned}
$$

Using MATLAB, a SIMULINK model is built and the desired trajectory input is obtained, Fig.4.

The hub rigid angle is chosen to be $\theta_{\mathrm{r}} \in[0, \pi]$ which normalized by using position factor P.F $\in[0,1]$. A smooth trajectory corresponds to the selected value of P.F is obtained by using a MATLAB code.

Figure 5 shows the joint angle $\theta_{r}[\mathrm{rad}]$ and the corresponding joint velocity and joint acceleration for two values of P.F .

For P. F $=0.1, \theta_{\text {rd }}=0.1 * 180=18^{\circ}$; Fig. 6 (a) represents desired velocity and acceleration of the joint angle. Figure 6(b) represents the joint and tip angle when applying the joint controller alone \& Fig. 6(c) represents the corresponding input torque to the flexible link model Fig. 6(d) shows the joint angles when using the DCLR and the MP-DCLR. \& Fig. 6(e) shows the corresponding input torques. Figure 6(f) shows the obtained tip angle for the three cases; the desired, with DCLR and with MP-DCLR. 


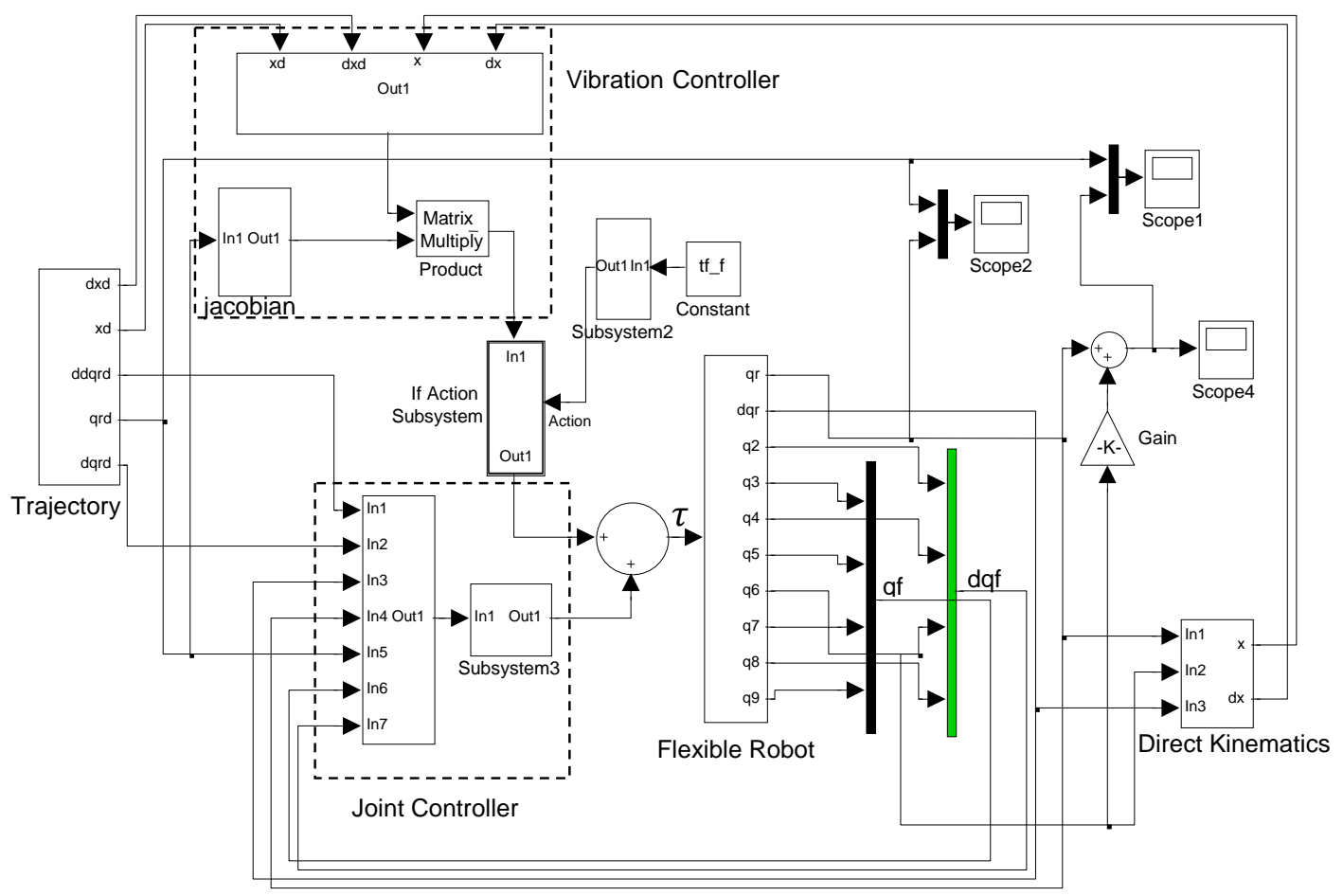

Fig. 4 SIMULINK model of flexible link manipulator with DCLR.
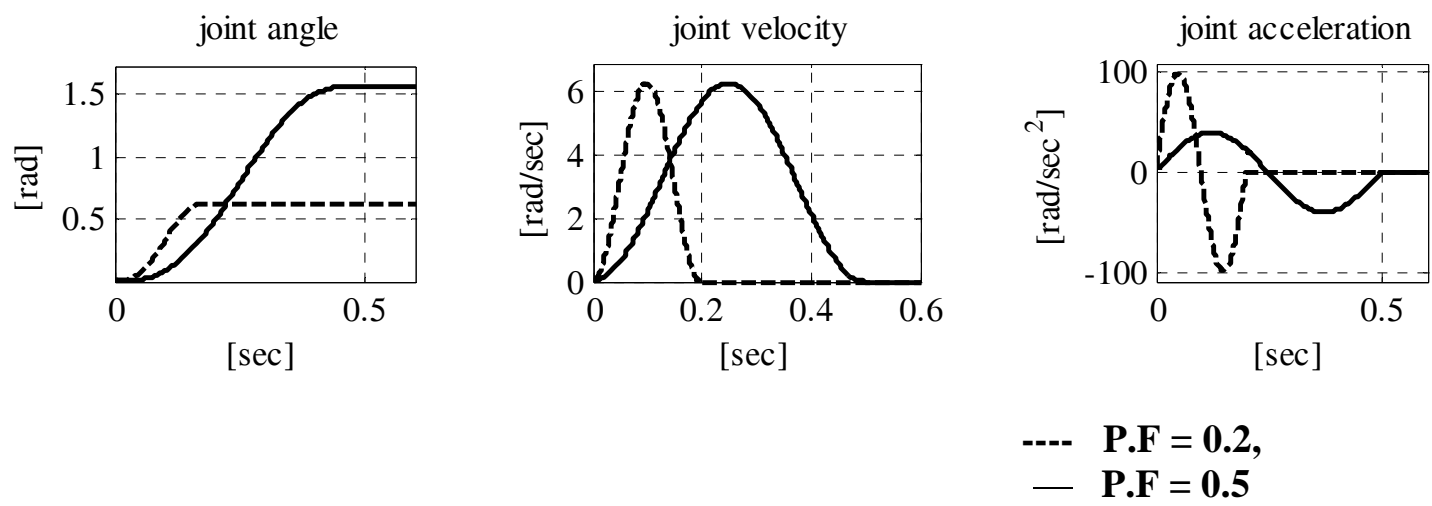

Fig. 5 Trajectory generated by previously written code.

Figure 7 shows the obtained tip angle for the same three cases in Fig. 6(f), but for P.F=0.1,0.3 and 0.5. Calculations are repeated for other different values of P.F and the obtained results are tabulated in Table (2) 

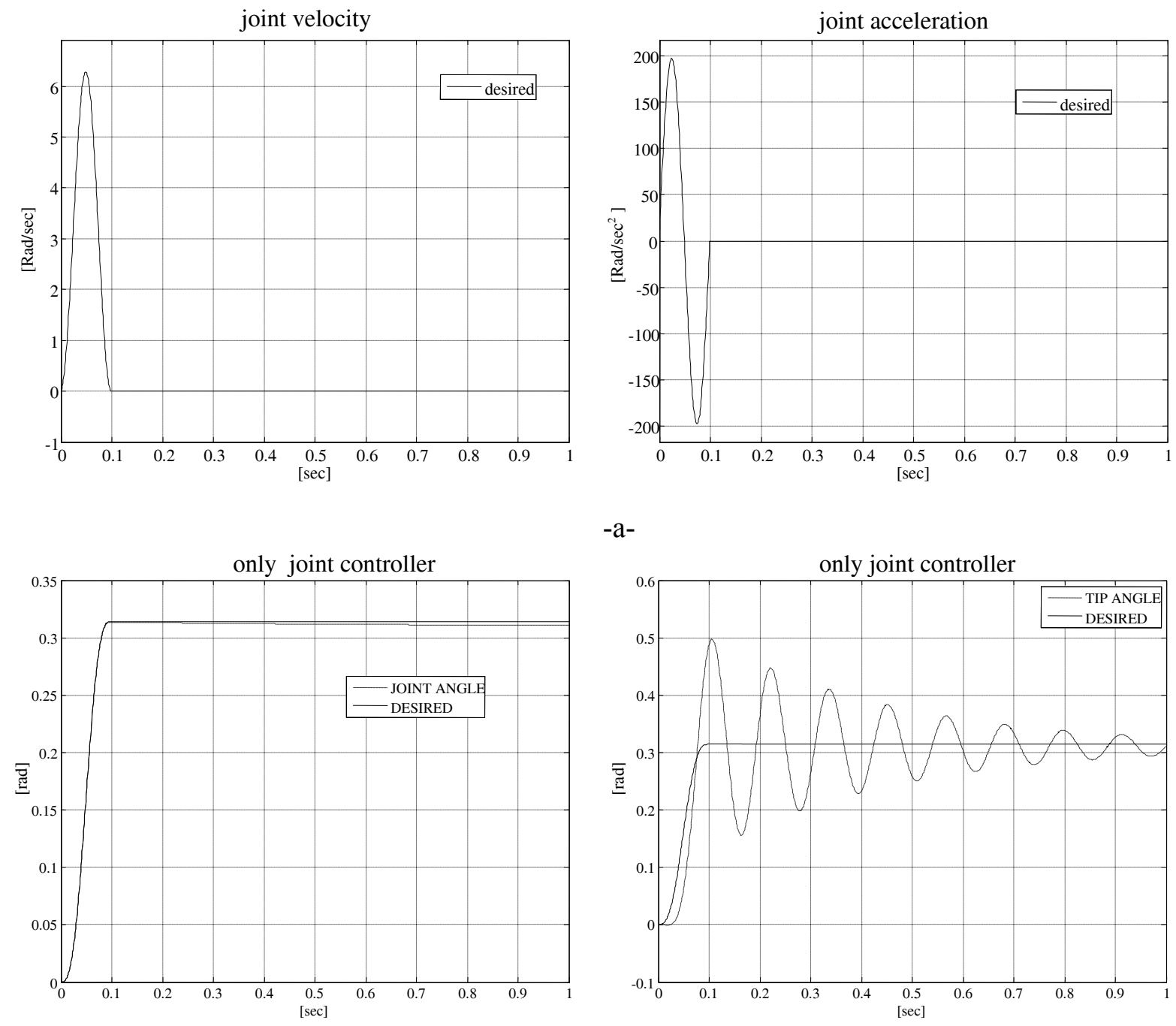

$-a-$

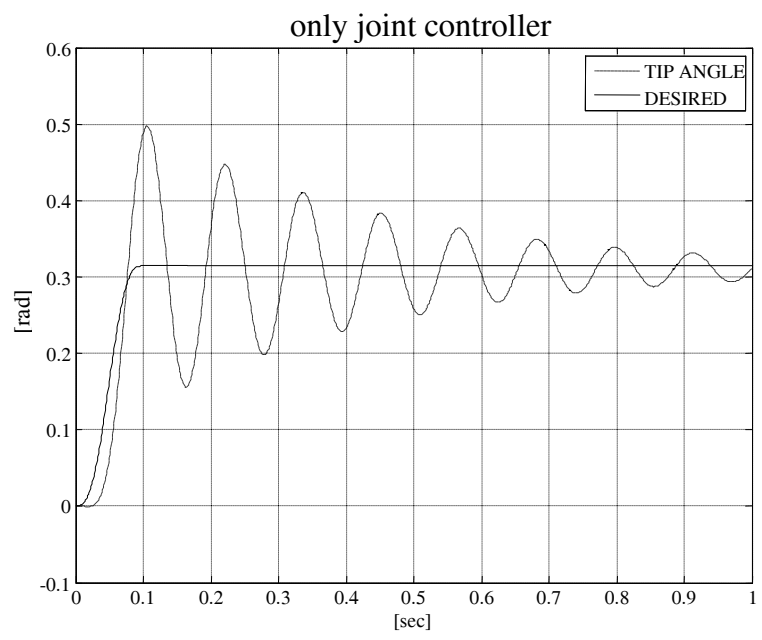

$-b-$

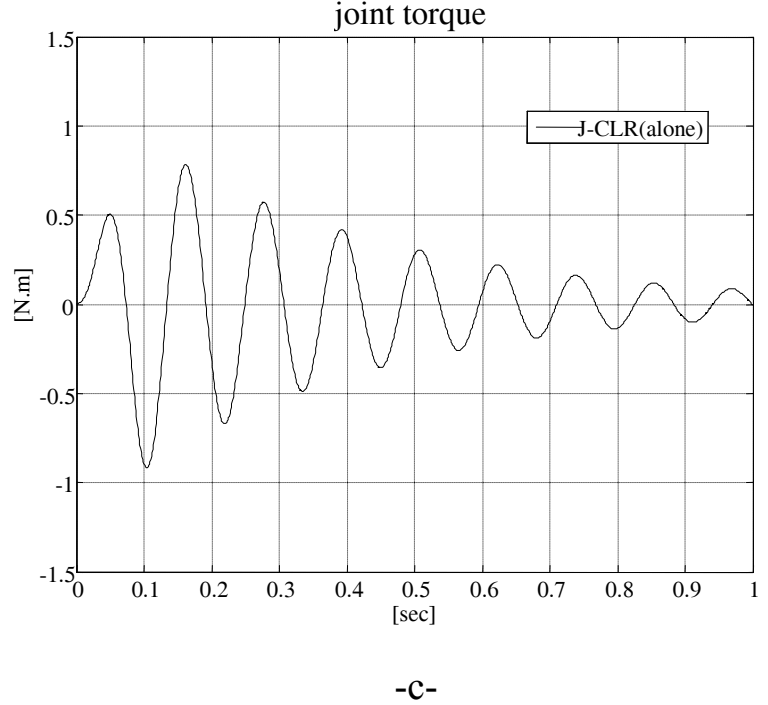

Fig. 6 Simulation outputs for $P . F=0.1$. 

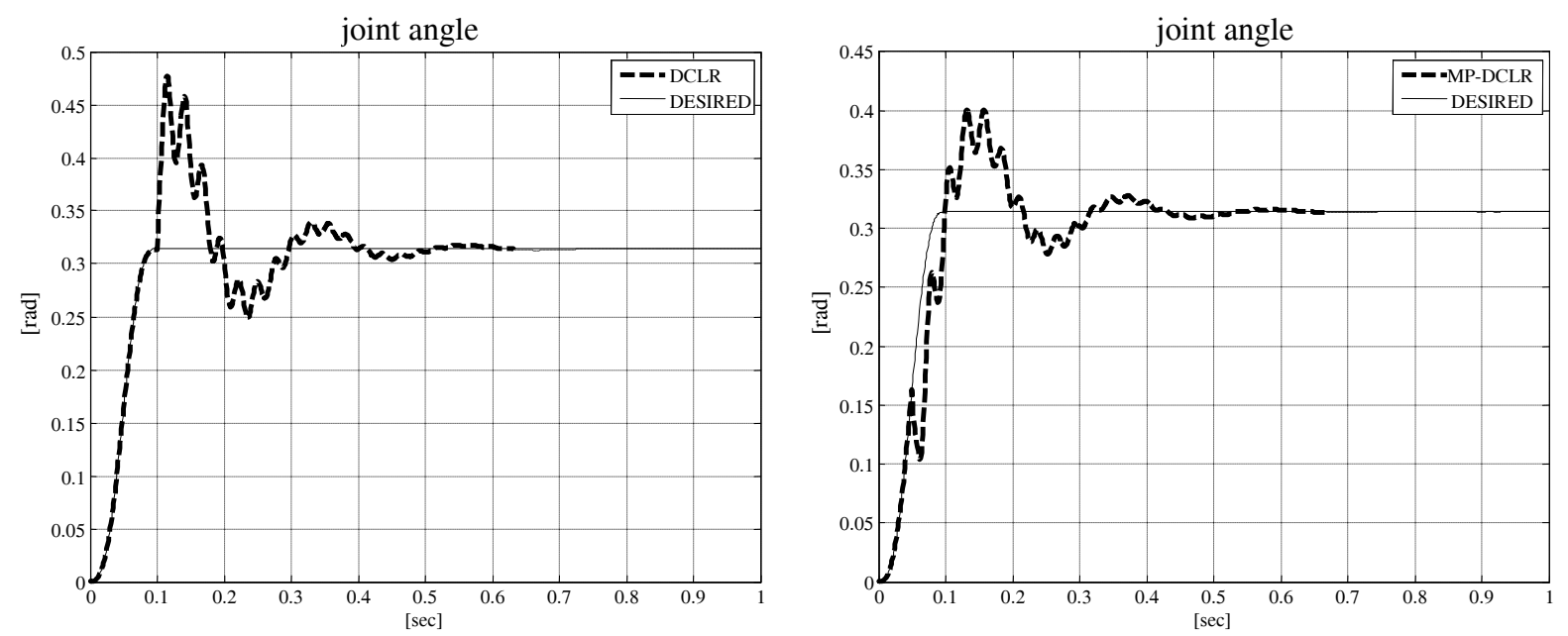

$-d-$
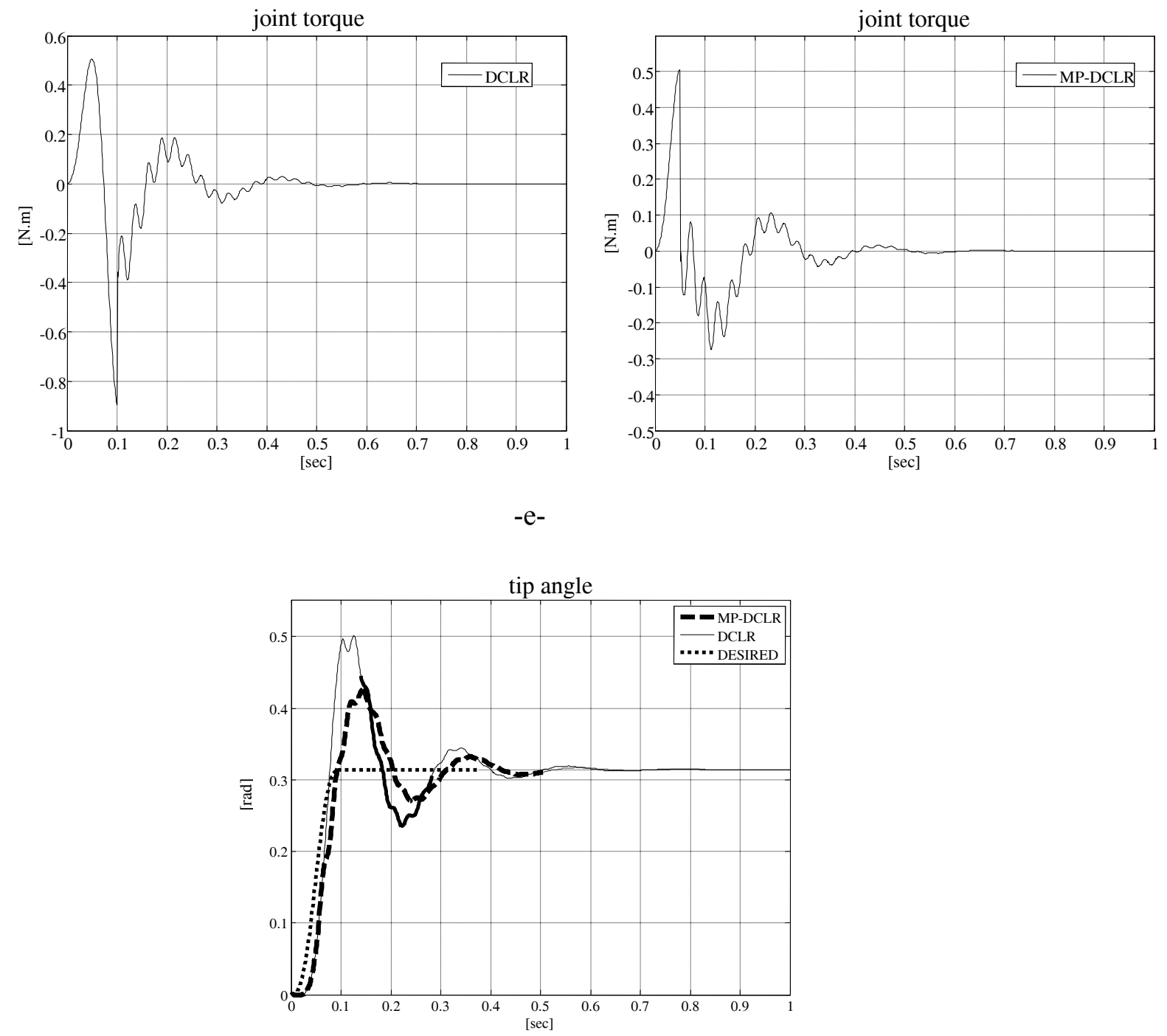

$-f-$

Fig. 6 (continued) Simulation outputs for $P . F=0.1$. 


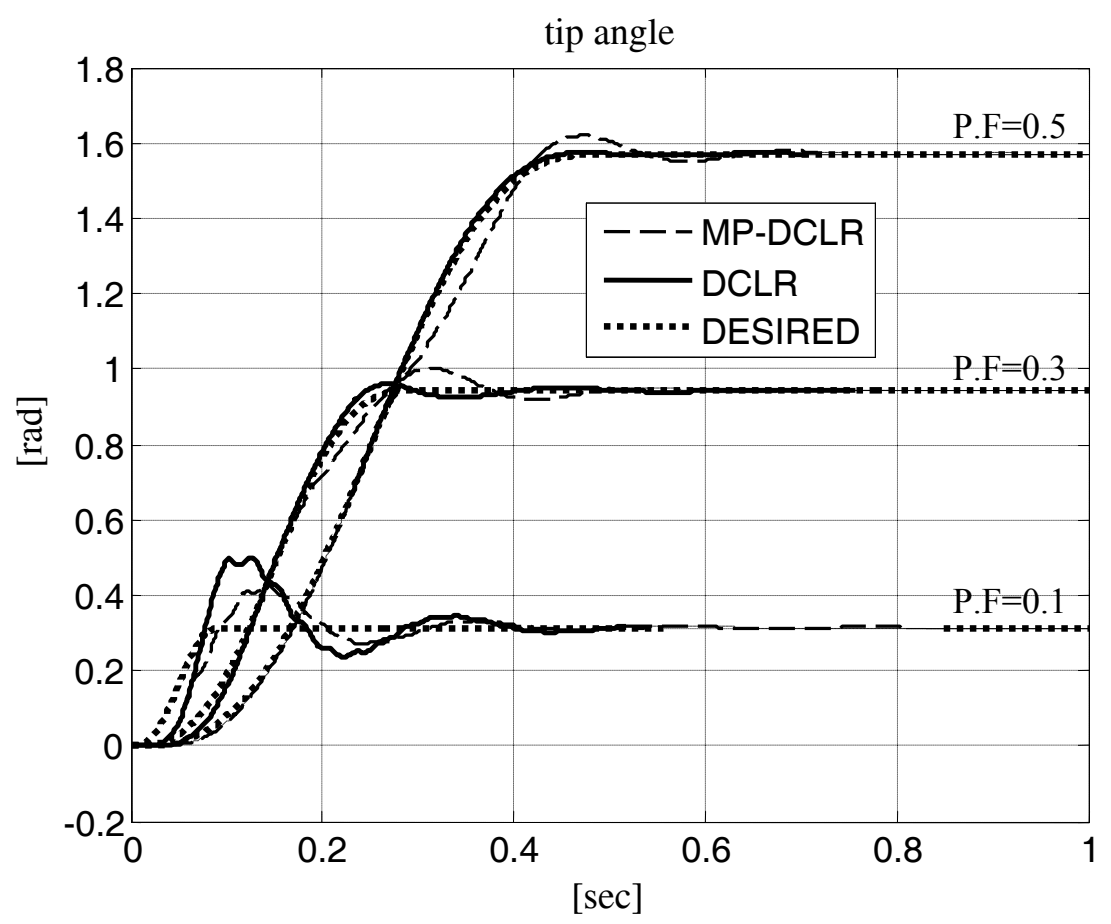

Fig. 7 Tip angle for P.F=0.1, P.F=0.3 and P.F=0.5 .

\section{Results and Conclusion}

Table 2, shows that for values of P.F less than 0.2 the MP-DCLR suppress the tip vibration effectively comparing with DCLR, while the case is different for higher values of PF. Simply for that robot under consideration it is recommended to use MP-DCLR for values of PF less or equal to 0.2 and DCLR for the rest of positions. The maximum $\&$ minimum tip deflections are $W_{\max }$ and $W_{\min }$ respectively. The subscript max is related to the case $\theta_{\text {tip }}>\theta_{r}$, while the subscript min is when $\theta_{\text {tip }}<\theta_{r} . W_{\max } / L$ represents the ratio between max deflection at tip point and length of link.

Table 2 Tip deflection comparison.

\begin{tabular}{|c|c|c|c|c|c|c|c|}
\hline \multirow[b]{2}{*}{ P.F } & \multirow[b]{2}{*}{ Acc. $\left(\mathrm{rad} / \mathrm{sec}^{2}\right)$} & \multicolumn{3}{|c|}{ MP-DCLR } & \multicolumn{3}{|c|}{ DCLR } \\
\hline & & \multicolumn{2}{|c|}{ Tip deflection W(m) } & $\mathrm{W}_{\max } / \mathrm{L}$ & \multicolumn{2}{|c|}{ Tip deflection W(m) } & $\mathrm{W}_{\max } / \mathrm{L}$ \\
\hline 0.10 & 197.39 & 0.031 & 0.024 & 0.077 & 0.031 & 0.054 & 0.176 \\
\hline 0.15 & 131.56 & 0.025 & 0.025 & 0.082 & .0248 & 0.042 & 0.138 \\
\hline 0.20 & 98.70 & 0.019 & 0.025 & 0.079 & 0.019 & 0.024 & 0.078 \\
\hline 0.25 & 78.95 & 0.015 & 0.020 & 0.066 & 0.015 & 0.011 & 0.037 \\
\hline 0.30 & 65.80 & 0.0116 & 0.0168 & 0.055 & 0.011 & $9.5 e-3$ & 0.037 \\
\hline 0.35 & 56.40 & $9.3 \mathrm{e}-3$ & 0.016 & 0.052 & $9.3 e-3$ & $8.6 e-3$ & 0.03 \\
\hline 0.40 & 49.40 & $7.6 \mathrm{e}-3$ & 0.0166 & 0.054 & $7.6 \mathrm{e}-3$ & $6.4 \mathrm{e}-3$ & 0.025 \\
\hline 0.45 & 43.86 & $6.3 e-3$ & 0.0167 & 0.054 & $6.3 \mathrm{e}-3$ & $5.6 e-3$ & 0.020 \\
\hline 0.50 & 39.47 & $6.5 \mathrm{e}-3$ & 0.0162 & 0.053 & $5.3 e-3$ & $5.3 e-3$ & 0.017 \\
\hline
\end{tabular}




\section{References}

[1] W. J. Book, O. Maizzo-Neto, D. E. Whiteny, "Feedback control of two beam, two joint systems with distributed flexibility"," ASME Journal of Dynamics Systems Measurements and Control, , 1975, pp.424-431.

[2] C. M. Okley, R. H. Cannon, E.F., "Anatomy of an Experimental Two-link Flexible Manipulator under End Point Control" ", Proceeding of IEEE Conference on Decision and Control, 1990, pp. 507-513.

[3] P. T. Kotnic, S. Yurkovich, U. Ozguner, "Acceleration feedback for control of a flexible manipulator arm”, “Journal of Robotic and System” 1988, pp. 181-195.

[4] H. Bolandi and S. M. Esmaeilzadeh, "Adaptive Nonlinear Sensor Output feedback Control of a Flexible Robot Arm", ", International Conference on Computer and Electrical engineering” 2008, pp.639-647.

[5] Ashitava. G., Robotics Fundamental Concepts and Analysis, Oxford University, 2007, pp. 340-346. 